\title{
Pratiques de la production et d'exploitation des fourrages dans la Région des Plateaux au Togo
}

\author{
Kodjo Akonta DEWA KASSA ${ }^{1 *}$, Amen Yawo NENONENE², Larounga TCHANILEY ${ }^{2}$ et \\ Koffi KOBA ${ }^{2}$ \\ 'Institut de Conseil et d'Appui Technique (ICAT), \\ ${ }^{2}$ Laboratoire de Recherche sur les Agroressources et la Santé Environnementale (LARASE) ESA-UL \\ 01 BP. 1515 Lomé 01, Université de Lomé, Togo. \\ "Auteur correspondant, E-mail : dewakassa@gmail.com,Tel : (00228) 90877620
}

\section{RESUME}

Avec la diminution progressive des surfaces pâturables liée à une multitude de facteurs dont le surpâturage, les aléas climatiques doublés des diverses activités anthropiques, l'élevage des ruminants se tourne de plus en plus vers la production fourragère afin d'assurer aux animaux une alimentation convenable et continue. Le but de cette étude est d'établir un état des lieux des pratiques de production et d'exploitation de fourrage effectuées par les éleveurs au Togo. A cet effet, 133 unités d'élevage de ruminants régulièrement suivies par l'Institut de Conseil et d'Appui Technique (ICAT), ont été enquêtées puis suivies pendant deux ans. Des résultats obtenus, il ressort que les espèces Gliricidia sepium, Panicum maximum et Leucaena leucocephala sont les fourrages les plus utilisés dans des proportions respectives de 92, 78 et $51 \%$. Aussi, l'utilisation des espèces fourragères dans l'installation des clôtures de maisons et d'habitats pour animaux, l'installation définitive de parcelle fourragère ainsi que l'insertion de la production fourragère dans la rotation culturale sont trois modes de production fourragère identifiés aux taux respectifs de 48, 31 et 15\%. Par ailleurs, l'affouragement au parc et la conduite sur parcelle fourragère sont essentiellement les deux méthodes d'exploitation des fourrages produits dans des proportions respectives de 62 et $37 \%$.

(C) 2018 International Formulae Group. All rights reserved.

Mots clés: fourrage, exploitation, Panicum maximum, Gliricidia sepium, Leucaena leucocephala.

\section{Forages production and exploitation practices in Plateaux Region of Togo}

\begin{abstract}
With the progressive reduction in pasturable surfaces related to a multitude of factors whose overgrazing, the doubled climatic risks of the various anthropic activities, the breeding of the ruminants turns more and more to the fodder production in order to ensure the animals a suitable and continuous food. The goal of this study is to establish an inventory of fixtures of the practices of production and exploitation of fodder carried out by the stockbreeders in Togo. To this end, 133 units of breeding of ruminants regularly followed by the Institute of the Council and Technical Support (ICAT), were surveyed then followed during two years. Results obtained, it arises that the species Gliricidia sepium, maximum Panicum and Leucaena leucocephala are the fodder most used in respective proportions of 92, 78 and $51 \%$. Also, the use of the fodder species in the installation of the fences of houses and habitats for animals, the final installation of fodder piece as well as the insertion of the fodder production in farming rotation are the three modes of fodder production identified at the respective rates of 48,31 and $15 \%$. In addition, the foddering with the park and control on fodder piece are primarily the two mining methods of the fodder produced in respective proportions of 62 and $37 \%$.
\end{abstract}

(C) 2018 International Formulae Group. All rights reserved.

Keywords: forage, exploitation, Panicum maximum, Gliricidia sepium, Leucaena leucocephala. 


\section{INTRODUCTION}

L'un des défis majeurs que la plupart des pays de l'Afrique de l'Ouest se sont fixés à relever, est de parvenir à assurer un niveau de sécurité alimentaire adéquat à leur population de plus en plus croissante. Avec le Programme National d'Investissement Agricole et de Sécurité Alimentaire (PNIASA), le Togo compte sur le secteur de l'agriculture en général et particulièrement sur le sous-secteur de l'élevage pour relever ce défi.

Au cours de ces dernières années,

l'élevage au Togo a été marqué par un accroissement des effectifs du cheptel (bovins, ovins, caprins) et de la population des éleveurs (Talaki, 2003). Cet accroissement est lié à deux facteurs essentiels. D'une part, il s'agit des grandes actions du Projet d'Appui au Secteur Agricole (PASA), un projet du PNIASA. Ces actions sont : la recapitalisation du cheptel, la maîtrise des maladies de grande incidence et l'amélioration des techniques d'élevage. D'autre part, cet accroissement est associé à l'explosion démographique qui induit un besoin de plus en plus croissant en produits carnés. Ce qui entrâne naturellement une augmentation des besoins en fourrage (Dewa Kassa, 2013).

Au même moment que les besoins en fourrage augmentent, on assiste à une rapide extension des surfaces cultivées (toujours en rapport avec la démographie galopante). L'action conjuguée de ces différents facteurs couplés à des épisodes de sécheresse, obligent souvent les éleveurs à opter pour le déplacement de leurs troupeaux vers des zones écologiques plus favorables (Kiema, 2002 ; Dewa Kassa, 2008 ; Sawadogo et al., 2012). Malheureusement, ces déplacements entrainent le plus souvent des conflits entre éleveurs et agriculteurs.

Face à cette situation, de nombreux éleveurs tentent de trouver sur place les voies et moyens nécessaires pour nourrir leurs animaux. A ce titre, de nombreux efforts ont été déployés pour la production et la conservation du fourrage en saison sèche (Sawadogo et al., 2012). Aussi, la complémentation de fourrage herbacé à base des ligneux, accessoire à une partie de l'année, devient indispensable en saison sèche chaude pour l'entretien des animaux (Bechir et al., 2009).

Le but de cette investigation est de répertorier les espèces fourragères les plus utilisées et d'établir un état des lieux des diverses pratiques de production et d'exploitation de fourrage adoptées par les éleveurs dans la Région des Plateaux au Togo.

\section{MATERIEL ET METHODES MATERIEL}

\section{Cadre géographique}

La Région des Plateaux représente le cadre géographique de la présente étude.

Avec pour chef-lieu Atakpamé, la Région des Plateaux est située à $7^{\circ} 30^{\prime} 0^{\prime \prime}$ de latitude nord et à $1^{\circ} 10^{\prime} 0^{\prime}$ 'de longitude Est. Elle représente 29,6\% du territoire national. Elle est limitée au nord par la Région Centrale, au sud par la Région Maritime, à l'est par le Bénin et à l'ouest par le Ghana.

La région jouit d'un climat subéquatorial caractérisé par deux saisons sèches et deux saisons pluvieuses. La grande saison des pluies s'étend entre mars et juin. Elle est suivie de la petite saison sèche (de juillet à août). Ensuite vient la petite saison pluvieuse (de septembre à octobre) et enfin la grande saison sèche (de novembre à février). La pluviométrie moyenne tourne autour de $1000 \mathrm{~mm}$ d'eau par an. Toutefois, elle varie d'une zone à l'autre entre 800 et $1700 \mathrm{~mm}$ d'eau par an au cours de ces 25 dernières années.

La région est traversée par le fleuve Mono et ses affluents (Anié, Ogou, Ofé, etc.). 
Dans la partie Est (zone écologique II), les formations végétales dominantes sont des savanes boisées guinéennes plus ou moins arborées. On y trouve également des forêts claires et des forêts-galeries discontinues le long des principaux cours d'eau (Kokou et al., 1999). Dans la partie Ouest (zone écologique IV) correspondant à la partie méridionale des monts du Togo encore appelés unité de l'Atakora, se trouvent des plaines du Litimé et d'Ahlon, les plateaux Akébou, Akposso, Dayes et Kloto. Elle constitue le domaine des forêts humides et semi-décidues du Togo (Akpagana, 1989a et 1992b).

Le potentiel fourrager est fourni par une végétation exubérante en saisons des pluies et sur une grande partie des saisons sèches. L'agriculture constitue pour cette région un secteur de production très important sur lequel s'appuie le développement industriel, artisanal et commercial.

\section{Matériel technique}

Cette étude est essentiellement effectuée au moyen de fiches d'enquête et de guides d'entretien.

\section{METHODES \\ Echantillonnage et enquêtes}

La prospection et l'identification des différents sites d'élevage de ruminants nous ont permis de constituer un échantillon à travers la Région des Plateaux. Au total, 133 unités d'élevage de ruminants suivies par les techniciens de l'ICAT ont été enquêtées dans huit (08) préfectures de janvier à mars 2014. La Figure 1 montre les zones concernées par la présente étude.

\section{Visites d'observation}

Ces 133 unités d'élevage ont fait chacune l'objet de 10 visites pendant 24 mois (avril 2014 à avril 2016). Ces visites permettent de faire des observations sur : les espèces fourragères les plus produites par les éleveurs, les modes de production ainsi que les manières d'exploitation de ces fourrages produits.

\section{Méthode d'analyse des données}

Un dépouillement manuel des fiches d'enquête et de suivi a été réalisé. Les informations obtenues ont été groupées suivant les objectifs visés. Le tableur Excel et le logiciel SPSS12 nous ont permis d'effectuer le traitement statistique des données. Des fréquences relatives au niveau de l'échantillon ont été calculées par rapport aux centres d'intérêt de l'étude.

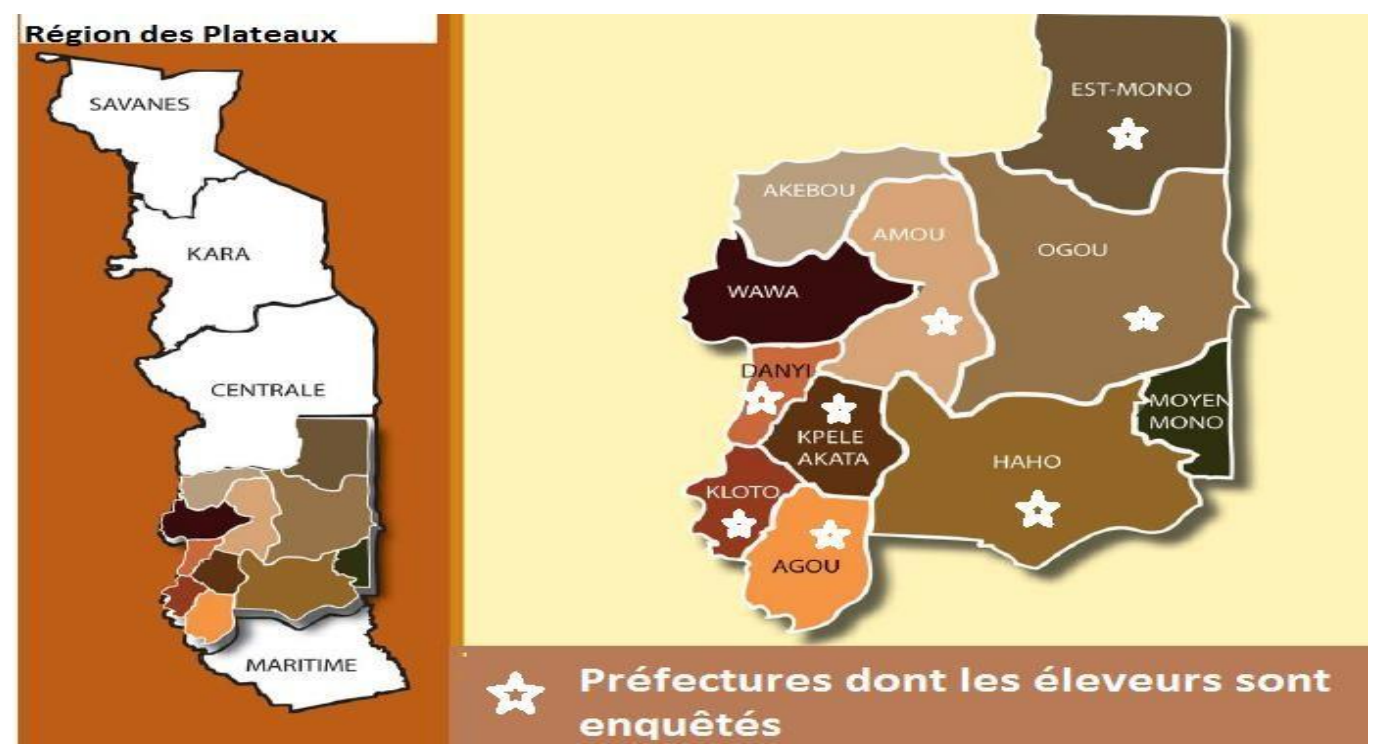

Figure 1: Carte de la localisation des Unités d'élevage enquêtées et suivies. 


\section{RESULTATS}

\section{Espèces fourragères produites}

Les espèces fourragères produites ont été répertoriées. Le Tableau 1 fait état des 03 espèces végétales les plus utilisées dans les pratiques de production fourragère dans les zones d'étude.

\section{Mode de production des espèces \\ fourragères}

Dans la région d'étude, parmi les différents modes de production de fourrage. identifiés, seuls 03 sont plus pratiquées (Tableau 2).

\section{Méthodes d'exploitation des espèces fourragères}

Trois méthodes d'exploitation des espèces fourragères sont essentiellement identifiées. Elles concernent l'affouragement au parc, la conduite au pâturage et l'alimentation au piquet dont les détails sont présentés dans le Tableau 3.

Tableau 1 : Espèces fourragères produites par les éleveurs dans les unités d'élevage (UE) enquêtées.

\begin{tabular}{|c|c|c|c|c|c|c|c|}
\hline \multirow{3}{*}{ Préfectures } & \multirow{3}{*}{$\begin{array}{l}\text { Nombre UE } \\
\text { enquêtées }\end{array}$} & \multicolumn{6}{|c|}{ Especes fourrageres } \\
\hline & & \multicolumn{2}{|c|}{ P. maximum } & \multicolumn{2}{|c|}{ G. sepium } & \multicolumn{2}{|c|}{ L. leucocephala } \\
\hline & & Nombre UE & Taux (\%) & Nombre UE & Taux (\%) & Nombre UE & Taux (\%) \\
\hline Agou & 20 & 18 & 90 & 18 & 90 & 9 & 45 \\
\hline Amou & 16 & 12 & 75 & 14 & 86 & 6 & 36 \\
\hline Dayes & 15 & 10 & 67 & 14 & 93 & 7 & 47 \\
\hline Est Mono & 15 & 13 & 87 & 13 & 87 & 8 & 53 \\
\hline Haho & 18 & 15 & 83 & 17 & 94 & 13 & 72 \\
\hline Kloto & 16 & 10 & 63 & 16 & 100 & 6 & 38 \\
\hline Kpélé & 16 & 11 & 69 & 14 & 86 & 8 & 50 \\
\hline Ogou & 17 & 15 & 88 & 17 & 100 & 11 & 68 \\
\hline TOTAL & 133 & 104 & - & 123 & - & 68 & - \\
\hline $\operatorname{Taux}(\%)$ & 100 & \multicolumn{2}{|l|}{78,20} & \multicolumn{2}{|c|}{92,48} & \multicolumn{2}{|c|}{51,13} \\
\hline
\end{tabular}

Tous ces pourcentages sont calculés par rapport au nombre d'UE enquêtées dans chacune des préfectures.

Tableau 2: Mode de production des espèces fourragères dans les unités d'élevage (UE) enquêtées.

\begin{tabular}{|c|c|c|c|c|c|c|c|}
\hline \multirow{3}{*}{ Préfectures } & \multirow{3}{*}{$\begin{array}{c}\text { Nb UE } \\
\text { enquêtées }\end{array}$} & \multicolumn{6}{|c|}{ Mode de production } \\
\hline & & \multicolumn{2}{|c|}{ Clôtures* } & \multicolumn{2}{|c|}{ Parcelles** } & \multicolumn{2}{|c|}{ Rotation culturale ${ }^{* * *}$} \\
\hline & & Nombre UE & Taux $(\%)$ & Nombre UE & Taux (\%) & Nombre UE & Taux (\%) \\
\hline Agou & 20 & 9 & 45 & 8 & 40 & 2 & 10 \\
\hline Amou & 16 & 8 & 50 & 4 & 25 & 3 & 19 \\
\hline Dayes & 15 & 8 & 53 & 4 & 26 & 2 & 13 \\
\hline Est Mono & 15 & 8 & 53 & 5 & 33 & 1 & 6 \\
\hline Haho & 18 & 7 & 39 & 6 & 33 & 4 & 22 \\
\hline Kloto & 16 & 9 & 56 & 5 & 31 & 2 & 13 \\
\hline Kpélé & 16 & 9 & 56 & 4 & 25 & 2 & 13 \\
\hline Ogou & 17 & 6 & 35 & 5 & 29 & 4 & 24 \\
\hline TOTAL & 133 & 64 & - & 41 & - & 20 & - \\
\hline Taux (\%) & 100 & \multicolumn{2}{|c|}{48,12} & \multicolumn{2}{|c|}{$\mathbf{3 0 , 8 3}$} & \multicolumn{2}{|c|}{15,04} \\
\hline
\end{tabular}

Clôture* = Utilisation des ligneux fourragers (G. sepium et L. leucocephala) pour la clôture des maisons et des enclos des animaux.

Parcelles** $=$ Utilisation des graminées fourragères $(P$. maximum) et quelques ligneux fourragers $(G$. sepium et $L$. leucocephala) dans la mise en place des parcelles fourragères.

Rotation culturale ${ }^{* * *}=$ Utilisation des légumineuses fourragères (C. Cajan) dans la rotation culturale. 
Tableau 3: Méthodes d'exploitation des espèces fourragères dans les unités d'élevage (UE) enquêtées.

\begin{tabular}{|c|c|c|c|c|c|c|c|}
\hline \multirow{3}{*}{ Préfectures } & \multirow{3}{*}{$\begin{array}{l}\text { Nombre UE } \\
\text { enquêtées }\end{array}$} & \multicolumn{6}{|c|}{ Mode d'exploitation } \\
\hline & & \multicolumn{2}{|c|}{ Affouragement au parc } & \multicolumn{2}{|c|}{ Conduite sur parcelle } & \multicolumn{2}{|c|}{ Alimentation au piquet } \\
\hline & & Nombre UE & Taux $(\%)$ & Nombr UE & Taux (\%) & Nombr UE & $\operatorname{Taux}(\%)$ \\
\hline Agou & 20 & 14 & 70 & 5 & 25 & 1 & 5 \\
\hline Amou & 16 & 11 & 69 & 5 & 31 & 0 & 0 \\
\hline Dayes & 15 & 10 & 67 & 5 & 33 & 0 & 0 \\
\hline Est Mono & 15 & 9 & 60 & 6 & 40 & 0 & 0 \\
\hline Haho & 18 & 11 & 61 & 7 & 39 & 0 & 0 \\
\hline Kloto & 16 & 8 & 50 & 8 & 50 & 0 & 0 \\
\hline Kpélé & 16 & 7 & 44 & 9 & 56 & 0 & 0 \\
\hline Ogou & 17 & 13 & 77 & 4 & 24 & 0 & 0 \\
\hline TOTAL & 133 & 83 & - & 49 & - & 1 & - \\
\hline Taux $(\%)$ & 100 & \multicolumn{2}{|c|}{62,41} & \multicolumn{2}{|c|}{36,84} & \multicolumn{2}{|c|}{0,75} \\
\hline
\end{tabular}

\section{DISCUSSION}

\section{Espèces fourragères produites}

Les espèces fourragères produites par les éleveurs dans les zones d'études sont liées à la qualité fourragère de celles-ci. L'indice de qualité des espèces herbacées prend en compte la période d'appétibilité de la plante, le degré d'appétibilité lié à l'anatomie et à la morphologie des feuilles et des tiges et la valeur fourragère (N'gom et al, Qualité pastorale des ressources herbagères de la réserve de biosphère du Ferlo, 2012 ; Int. J. Biol. Chem. Sci. 6(1): 186-201). La légumineuse ligneuse $G$. sepium est retrouvée dans $92 \%$ des élevages enquêtés suivie de l'espèce $P$. maximum $(78 \%)$ et de $L$. leucocephala $(51 \%)$. Au-delà de ces 03 espèces fourragères, on retrouve le mucuna $(31,15 \%)$, l'espèce Penniseutum purpureum (30,79\%), l'espèce Sorghum bicolore (30,35\%), l'espèce Spondias monbin $(30,27 \%)$ très utilisée comme plante thérapeutique, des espèces de Terminalia sp. $(15,07 \%)$ des espèces de Ficus sp. (06,35\%), Le Tableau 1 présente uniquement les proportions des 03 espèces les plus utilisées par les éleveurs de ruminants dans les UE enquêtées de la Région des Plateaux. Ces espèces qui sont respectivement le $G$. sepium, le $P$. maximum et le $L$. leucocephala sont produites respectivement dans des proportions de $92,48 \%, 78,20 \%$ et $51,13 \%$.

Par ailleurs, d'un côté, il convient de souligner qu'en matière de graminées fourragères utilisées dans les UE, l'espèce $P$. maximum $(92,48 \%)$ est de loin la graminée la plus produite devant l'espèce Penniseutum purpureum $(30,79 \%)$ et le Sorghum bicolore ((30,35\%), L'importance du choix de la graminée $P$. maximum serait liée à certaines de ses caractéristiques reconnues par les enquêtés. Il s'agit essentiellement de sa productivité en biomasse, sa résistance aux aléas climatiques notamment au stress hydrique, son appétence par les herbivores domestiques, sa résistance au feu de brousse, au broutage et au 
piétinement (Adjolohoun et al., 2012, 2013). Ce qui rejoint les motivations des essais menés par l'ORSTOM dans les années 1960 sur l'espèce $P$. maximum ayant abouti à la sélection des cultivars très productifs et de bonne valeur fourragère (Toutain et al., 2009).

D'autre part, il importe de préciser que parmi la multitude de légumineuses exploitées dans les UE de la zone d'étude, l'espèce $G$. sepium est de loin la plus produite par les éleveurs à cause de son excellente appétibilité, de sa résistance au stress hydrique et de l'importance de sa biomasse. Par contre, très appréciée dans un passé récent, l'espèce $L$. leucocephala est entrain de connaître une chute dans le choix des légumineuses produites par les éleveurs à cause de son effet envahisseur à travers la propagation non contrôlée de ses graines (ISSG, 2016). Toutefois, sa production est maintenue dans plus de la moitié des UE à la reconnaissance de ses qualités par les éleveurs à savoir : sa fréquence de reprise après la coupe, son appétibilité, sa résistance au stress hydrique et à la variabilité climatique, sa biomasse, etc. La résistance au stress hydrique de cette espèce comme celle de G. sepium serait en partie liée à leur système racinaire profond qui permet à ces plantes de puiser de l'eau à des profondeurs que ne pourront pas réaliser les espèces graminéennes (Adjolohoun et al., 2008).

Aussi, il faut souligner que dans $50 \%$ des préfectures enquêtées (Agou, Amou, Dayes et Kloto), le seuil de production de l'espèce $L$. leucocaphala est en dessous de 50\%. Dans les préfectures de Haho et de l'Ogou, l'espèce $L$. leucocaphala est encore produite à un seuil élevé (respectivement $72 \%$ et $65 \%$ ) dans les UE enquêtées. Toutefois, dans les deux préfectures, elle est relativement moins produite $(94,44 \%)$ par rapport à l'espèce $G$. sepium (100\%).

Par ailleurs, il convient de souligner que certains domaines fourragers sont fortement envahis par l'espèce Hyptis suaveolens. L'indice de qualité et le niveau d'invasion peuvent servir d'indicateurs essentiels de la qualité fourragère des parcours envahis en l'absence de toute analyse bromatologique (Oumorou et al., 2010).

\section{Mode de production des espèces fourragères}

En matière de production des espèces fourragères des UE ciblées par la présente étude, trois (03) modes sont essentiellement adoptés (Tableau 2). Premièrement, plus de $48 \%$ des UE installent les plantes fourragères de manière à servir de clôtures de maison ou d'habitat pour animaux (bergeries et chèvreries). Les espèces fourragères arbustives comme G. sepium, Spondias mombin sont les plus utilisées. Cette pratique est très courante dans toutes les préfectures de la zone d'étude.

Ensuite, près de $31 \%$ des UE ont installé des parcelles fourragères protégées à proximité des concessions afin d'éviter la divagation des animaux et leur garantir une alimentation convenable. En général, on y trouve des plantes telles que $P$. maximum, $G$. sepium, L. leucocephala, Cajanus cajan et Centrosema pubecsens. Les autres espèces telles que Spondias mombin, Vernonia amygdalina, Newbouldia laevis, Carica papaya, etc. sont plus ou moins faiblement représentées et sont exploitées à des fins thérapeutiques. Ce mode de production de fourrage est moyennement adopté dans l'ensemble des UE. Il convient de souligner que cette pratique est faiblement adoptée dans 03 préfectures qui sont : Kpélé (25\%), Amou $(26,67 \%)$ et Ogou $(29,41 \%)$.

Enfin, 15\% des UE ciblées introduisent la production de fourrage dans la rotation des cultures. Les éleveurs concernés par cette pratique, installent des essences fourragères en fin de cycle des cultures vivrières. Les espèces les plus utilisées dans cette pratique sont entre autre : Cajanus cajan, Centrosema pubescens, Sorghum bicolore et $P$. maximum. Ces espèces plantées sont soutenues par la végétation naturelle qui reprend une fois que la parcelle est mise en jachère. Il faut préciser que cette pratique est très opérationnelle dans les préfectures de : Ogou, Haho et Amou respectivement à des taux de $23,53 \% ; 22,22 \%$ et $18,75 \%$. Contrairement à ces préfectures, la préfecture de l'Est-Mono connait un taux très bas $(6,67 \%)$ dans l'adoption de ce mode de production fourragère. 
Par ailleurs, au-delà de ces trois (03) modes sus-indiqués, 02 autres sont identifiés dans $6 \%$ de l'ensemble des UE enquêtées. Il s'agit de l'installation en vrac des espèces fourragères dans un espace plus ou moins vaste $(2,26 \%)$ et la conservation d'une partie de la végétation naturelle riche $(3,76 \%)$.

\section{Méthodes d'exploitation des espèces fourragères}

En termes de méthodes d'exploitation des espèces fourragères produites dans les UE concernées par cette étude, outre la divagation (pratique courante) qui est observée dans plus de $90 \%$ des unités d'élevage en saison sèche et une partie des saisons pluvieuses, l'affouragement au parc et la conduite des animaux au pâturage sont remarquablement les deux autres méthodes pratiquées dans les proportions respectives de plus de $62 \%$ et près de $37 \%$. Il est important de souligner que ces deux méthodes sont chacune relativement plus pratiquée dans certains milieux que dans d'autres. Ainsi, l'affouragement au parc est plus pratiqué dans les préfectures de : Ogou (76,47\%), Agou (70\%), Amou $(68,75 \%)$ et Dayes $(66,67 \%)$. De la même façon, la conduite au pâturage est plus pratiquée dans les préfectures de : Kpélé $(56,25 \%)$, Kloto (50\%), Est Mono (40\%) et Haho (38,89\%).

Par ailleurs, il faut préciser que l'alimentation au piquet est moins pratiquée à cause de sa complexité avec les UE à effectif important. Elle se pratique le plus souvent par des éleveurs qui ne disposent qu'un effectif relativement réduit de bétail.

\section{Conclusion}

Le présent travail a consisté à faire un état des lieux des diverses pratiques de production et d'exploitation des fourrages dans la Région des Plateaux au Togo.

Au total, 133 Unités d'élevage suivies chacune 10 fois pendant 24 mois ont fait l'objet d'observations sur les espèces les plus produites par les éleveurs, les modes de production ainsi que les manières d'exploitation de ces fourrages produits.

Il ressort des résultats que les espèces les plus produites sont: G. sepium $(92,48 \%), P$. maximum $(78,20 \%)$ et $L$. leucocephala $(51,18 \%)$.
En matière de mode de production de ces espèces fourragères, on note : l'utilisation des ligneux (G. sepium et L. leucocephala) dans l'installation des clôtures des maisons et des enclos pour animaux $(48,12 \%)$; l'utilisation des graminées fourragères ( $P$. maximum) et quelques ligneux fourragers ( $G$. sepium et $L$. leucocephala) dans l'installation des parcelles fourragères protégées à proximité des concessions afin d'éviter la divagation des animaux et leur garantir une alimentation convenable $(30,33 \%)$ puis l'utilisation des légumineuses fourragères (Cajanus cajan) dans la rotation culturale $(15,04 \%)$.

S'agissant de mode d'exploitation des espèces fourragères produites, on note l'affourragement au parc $(62,41 \%)$ et la conduite sur parcelle fourragère (36 ?84\%).

\section{REMERCIEMENTS}

Nous remercions très sincèrement le PPAAO-Togo pour son appui financier, l'ICAT pour son appui technique et l'ESA pour son appui multiforme. Nos pensées vont également à l'endroit de tous ceux qui nous ont soutenus et apporté leur concours dans la recherche d'informations et dans l'exécution du présent travail.

\section{REFERENCES}

Adjolohoun S. 2008. Yield, nutritive value and effects on soil fertility of forage grasses and legumes cultivated as ley pastures in the Borgou region of Benin. Thesis, Gembloux, Belgium, 101p.

Adjolohoun S, Dahouda M, Adandédjan C,

Toleba SS, Houinato M, Nonfon R,

Sinsin B. 2012. Diversité et caractérisation morphologique des écotypes de l'espèce fourragère Panicum maximum au Bénin. Int. J. Biol. Chem. Sci., 6(5): 1838-1852.

Adjolohoun S, Dahouda M, Adandedjan C, Toleba SS, Kindomihou V, Sinsin B. 2013. Evaluation of biomass production and nutritive value of nine Panicum maximum ecotypes in Central region of Benin. African Journal of Agricultural Research, 8(17): 1661-1668.

Akpagana K. 1989a. Recherches sur les forêts denses humides du Togo. Th. Doct. Sc. Nat., Univ. Bordeaux III, France, 195p. 
Akpagana K. 1992b. Espèces nouvelles pour la flore du Togo. Ann. Univ. Bénin, sect. sci., 10 : pp. 25-32.

Bechir AB, Mopate 1 LY, Kabore-Zoungrana CY. 2009. Evaluation de la disponibilité saisonnière du fourrage ligneux en zone soudanienne du Tchad: cas du terroir de N'Guetté 1. Int. J. Biol. Chem. Sci., 3(1): 135-146.

Claude J, Grouzis M, Milleville P. 1991. Un Espace Sahélien : la Mare d'Oursi (Burkina Faso). Editions de l'ORSTOM : Paris ; 254p.

Dewa Kassa KA. 2008. La transhumance interEtats au Togo : Causes, organisation et conséquences au Togo. Mémoire d'Ingénieur Agronome, ESA-UL, 105 p.

Dewa Kassa KA. 2013. Etude de la croissance de deux variétés de Panicum maximum et effets de leur association avec deux espèces de légumineuses, Mém. DEA SAGE. Univ. Lomé 36p.

Gù-Konu EY, Laclavère G. 1981. Atlas du Togo, Editions JA, Les Atlas Jeune Afrique ; 1349p.

Kiema A. 2002. Ressources pastorales et leurs modes d'exploitation dans deux terroirs sahéliens au Burkina Faso. Mémoire de DEA. Institut Polytechnique de Bobo Dioulasso, Burkina Faso, p66.
Kokou K, Afidégnon D, Guelly KA, Roussel B, Akpagana K. 1999. Dynamique de la végétation périphérique du barrage hydroélectrique de Nangbéto sur le fleuve Mono (Togo) après la mise en eau. Espace, Culture et Développement dans la région d'Atakpamé. pp.103-112.

Ngom D, Bakhoum A, Diatta S, Akpo LE. 2012. Qualité pastorale des ressources herbagères de la réserve de biosphère du Ferlo (Nord-Sénégal). Int. J. Biol. Chem. Sci., 6(1): 186-201.

Oumorou M, Aboh BA, Babatounde S, Houinato M, Sinsin B. 2010 - Valeur pastorale, productivité et connaissances endogènes de l'effet de l'invasion, par Hyptis suaveolens L. Poit., des pâturages naturels en zone soudano-guinéenne (Bénin). Int. J. Biol. Chem. Sci., 4(4): 1262-1277.

Sawadogo I, Devineau JL, Fournier A. 2012.

Etat des ressources pastorales dans une terre d'accueil et de transit des pasteurs transhumants : le terroir de Kotchari (sudest du Burkina Faso). La Terre et la VieRevue d'Ecologie, 67(2) : 157-178.

Talaki E. 2013. Revue des filières bétail/viande, lait et des politiques qui les influencent au Togo. FAO et ECOWAS; $71 \mathrm{p}$.

Toutain B, Klein HD, Lhoste P, Duteurtre G. 2009. Histoire et avenir des cultures fourragères en Afrique tropicale. CIRAD ; $13 p$. 